\title{
Automated ambiguity estimation for VLBI Intensive sessions using L1-norm
}

\author{
Niko Kareinen, Thomas Hobiger, Rüdiger Haas \\ Chalmers University of Technology \\ Department of Earth and Space Sciences \\ SE-412 96 Gothenburg \\ Sweden
}

\section{Abstract}

Very Long Baseline Interferometry (VLBI) is a space-geodetic technique that is uniquely capable of direct observation of the angle of the Earth's rotation about the Celestial Intermediate Pole (CIP) axis, namely UT1. The daily estimates of the difference between UT1 and Coordinated Universal Time (UTC) provided by the 1-hour long VLBI Intensive sessions are essential in providing timely UT1 estimates for satellite navigation systems and orbit determination. In order to produce timely UT1 estimates, efforts have been made to completely automate the analysis of VLBI Intensive sessions. This involves the automatic processing of X- and S-band group delays. These data contain an unknown number of integer ambiguities in the observed group delays. They are introduced as a side-effect of the bandwidth synthesis technique, which is used to combine correlator results from the narrow channels that span the individual bands. In an automated analysis with the c5++ software the standard approach in resolving the ambiguities is to perform a simplified parameter estimation using a least-squares adjustment (L2-norm minimisation). We implement L1-norm as an alternative estimation method 
in $\mathrm{c} 5++$. The implemented method is used to automatically estimate the ambiguities in VLBI Intensive sessions on the Kokee-Wettzell baseline. The results are compared to an analysis set-up where the ambiguity estimation is computed using the L2-norm. For both methods three different weighting strategies for the ambiguity estimation are assessed. The results show that the L1-norm is better at automatically resolving the ambiguities than the L2-norm. The use of the L1-norm leads to a significantly higher number of good quality UT1-UTC estimates with each of the three weighting strategies. The increase in the number of sessions is approximately $5 \%$ for each weighting strategy. This is accompanied by smaller post-fit residuals in the final UT1-UTC estimation step.

Keywords: Earth rotation, UT1, VLBI, automated analysis, robust estimation

\section{Introduction}

Very Long Baseline Interferometry (VLBI) is a unique technique among space-geodetic techniques due to its capability to determine all Earth Orientation Parameters (EOP) simultaneously. These parameters provide the orientation of the Earth in an inertial reference system. One of the parameters is the Earth's rotation about the Celestial Intermediate Pole (CIP) axis, which is described as Universal Time (UT1). VLBI measures the difference between the UT1 and Universal Coordinated Time (UTC), UT1-UTC, from which the UT1 can subsequently be estimated.

By monitoring Earth rotation it is possible to gather information about the underlying geodynamical behaviour of the Earth system. Thus, also UT1 
as a parameter is connected to various geophysical phenomena, in particular via the exchange of angular momentum between the atmosphere, geophysical fluids, and the solid earth (Barnes et al., 1983). Thus, high-frequency signals in UT1 can be used to study these geophysical excitations and the underlying geodynamical phenomena (Brzeziński, 2012). Moreover, the impact of earthquakes with large magnitude, such as the Denali earthquake in 2002, has also been verified by EOP parameters (Titov and Tregoning, 2005), and therefore stresses the need for real-time EOP monitoring.

Furthermore, timely UT1 estimates from VLBI are crucial for spacegeodetic techniques such as Global Satellite Navigation Systems (GNSS). GNSS are only capable of accessing UT1 via its time derivative, usually denoted as the change in Length-of-Day (LOD), and rely on UT1 input from VLBI.

The International VLBI Service for Geodesy and Astrometry (IVS) (Behrend, 2013) organises daily 1-hour long VLBI observing sessions called the Intensive sessions (INT). Characteristic to these sessions is that they are observed on extended East-West-baselines using a network of 2 to 3 antennas. Currently three types of INT sessions are conducted regularly. INT1 are observed on the Kokee (Hawaii) - Wettzell (Germany) baseline from Monday to Friday at 18:30 UTC. INT2 are observed on the Tsukuba (Japan) Wettzell (Germany) baseline on Saturday and Sunday at 7:30 UTC. Finally, to fill in the gap between the last INT2 of the week and the first INT1, INT3 are observed with a network consisting of Wettzell, Tsukuba, and Ny-Ålesund on Monday mornings at 7:00 UTC. The short duration of the sessions and the baseline geometry leads to a relatively low number of approximately 20- 
40 observations per baseline. The aim of the INT sessions is to produce daily UT1 estimates in a timely fashion. The turnaround time of the results depends on the VLBI processing chain. Namely, the time it takes to correlate the observed data to produce databases, which are subsequently analysed by various VLBI analysis packages in order to obtain the UT1 estimates. One way to streamline this analysis chain is to automatically process the correlated data. Automated near-real time analysis of INT sessions has been investigated in e.g. Hobiger et al. (2010) and Kareinen et al. (2015).

Geodetic VLBI sessions are typically observed on two frequency bands centred around $8.4 \mathrm{GHz}$ (X-band) and $2.3 \mathrm{GHz}$ (S-band). A linear combination of the observed delays on the two bands can be used to derive a delay observable that is almost completely free of ionospheric effects. The two bands consist of individual channels, which are in the post-correlation procedure combined with a bandwidth synthesis technique (Rogers, 1970) to span the whole bandwidth. A side-effect of this procedure is that an unknown number of integer ambiguities are introduced into the observed group delays. The ambiguities are proportional to the channel spacing within the individual bands. For a typical channel set-up in an INT session these ambiguities are 50 ns for X-band and 200 ns for S-band. For comparison, the formal errors for the observed delays in the correlator output for the INT sessions are approximately three orders of magnitude smaller. Before the ionospheric calibration can be computed, the ambiguities have to be resolved on each band. Any unresolved ambiguities in the observed group delays will propagate into the UT1 estimates.

There are multiple available VLBI software packages which can be used 
to estimate geodetic parameters from VLBI observations. These include e.g. c5++ (Hobiger et al., 2010), CALC/SOLVE (Ma et al., 1990), Vienna VLBI Software (VieVS) (Böhm et al., 2012), GEOSAT (Andersen, 2000), OCCAM (Titov et al., 2004), and OCCAM/GROSS (Malkin and Skurikhina, 2005). A recent modernisation for the SOLVE part in CALC/SOLVE is $v$ Solve (Bolotin et al., 2014). Out of these software packages, c5++, CALC/SOLVE, and $v$ Solve are the only ones that allow to resolve the group delay ambiguities to produce ambiguity- and ionosphere-free X-band databases. The databases produced by the correlator contain group delays which include ambiguities and ionospheric effects. These databases are referred to as Version-1 databases.

The standard approach for parameter estimation in all software packages mentioned above is the method of least-squares adjustment (Koch, 1999) (i.e. L2-norm minimisation). In this paper, as an alternative approach to the L2-norm, we implement parameter estimation based on the L1-norm and apply it to the analysis of the INT sessions in the ambiguity resolving step. Furthermore, we evaluate alternative weighting strategies for both the L1and L2-norm ambiguity estimation. Compared to the L2-norm, the L1-norm should be more robust in the presence of outliers. We investigate whether this robust estimator helps to correctly detect the ambiguities in the initial stages of the analysis process. Starting from Version-1 databases we use the modified c5++ to automatically analyse INT1 sessions from 2001 to 2015 to estimate UT1. 


\section{Formulation of the minimisation conditions}

The objective functions for both the L1- and L2-norm minimisations can be derived from the general expression for a p-norm, which is given by

$$
\|x\|_{p}=\left(\sum_{i=1}^{p}\left|x_{i}\right|^{p}\right)^{\frac{1}{p}} .
$$

The L1-norm and L2-norms correspond to Equation 1 with p-values of $\mathrm{p}=1$ and $\mathrm{p}=2$, respectively. In both cases the norms to be minimized are functions of the residuals $v_{i}$ between the functional model and the observations, as well as possible weighting terms. The weight terms are included as multiplicative factors in the summands of the norms. Thus for, L1 and L2 the objective functions to be minimised are given respectively by

$$
\begin{aligned}
& \mathrm{L} 1: \min \left(\mathbf{p}^{\top}|\mathbf{v}|\right), \\
& \mathrm{L} 2: \min \left(\mathbf{v}^{\top} \mathbf{P} \mathbf{v}\right),
\end{aligned}
$$

where $\mathbf{v}$ is a vector containing the residuals for $n$ observations, $\mathbf{p}$ is a vector containing the associated weights for the observations, and $\mathbf{P}$ is an $n_{\text {obs }} \times n_{\text {obs }}$ matrix in which the diagonal contains the weights for the observations and the off-diagonal elements the possible correlation terms.

In the following subsections, first the standard L2-norm minimisation procedure is described. Then the derivations of the equations needed to solve the L1-norm minimisation problem are discussed.

\subsection{L2-norm minimisation}

A detailed description of the L2-norm minimisation can be found in e.g. Koch (1999). Generally, the L2-norm minimisation is done according to the 
condition given by Equation 3. A linear functional model in matrix form is given by

$$
\mathbf{v}=\mathbf{A x}-\mathbf{y}
$$

where $\mathbf{v}$ is the residual vector, $\mathbf{A}$ is the design matrix, $\mathbf{x}$ is the vector containing the unknown parameters of the model, and $\mathbf{y}$ is the observation vector. The design matrix $\mathbf{A}$ contains information on how the unknown variables relate to the observations in the functional model. Often the initial functional model of the system in question is not linear. In this case the system needs to be linearised. In a linearised system the design matrix will contain the partial derivatives of the model with respect to the unknown parameters. Using the expression for the residuals given by Equation 4 in Equation 3, we can write the weighted sum of the residuals as

$$
\mathbf{v}^{\top} \mathbf{P} \mathbf{v}=(\mathbf{A x}-\mathbf{y})^{\top} \mathbf{P}(\mathbf{A x}-\mathbf{y})
$$

Differentiating the expression in Equation 5 with respect to $\mathbf{x}$ and setting it to equal 0 we obtain

$$
\hat{\mathbf{x}}=\left(\mathbf{A}^{\top} \mathbf{P A}\right)^{-1} \mathbf{A}^{\top} \mathbf{P} \mathbf{y}
$$

From Equation 6 we obtain the vector of unknowns, which will minimise the squared sum of the weighted residuals. An important property of the L2-norm is that the absolute value term in the sum is squared, and thus the absolute value function can be omitted. This enables us to differentiate the expression given in Equation 5. Thus, the L2-norm is computationally straightforward, with the most costly operation being the matrix inversion 
in Equation 6. In the case of the L1-norm the differentiability is an issue and requires an alternative approach. The standard parameter estimation in the $\mathrm{c} 5++$ analysis software is based on iterative least-squares (L2-norm) minimisation.

\subsection{L1-norm minimisation}

The L1-norm minimisation, which is discussed in detail in e.g. Koch (1999), starts from the same functional model set-up used in Equation 4. However, now the residual vector $\mathbf{v}$ remains inside the absolute value function. Consequently, it is not differentiable at 0 , and we are unable to derive the value for the vector of unknowns $\mathbf{x}$ that will minimise the sum of the weighted absolute values of the residuals. The formulation for a L1-norm minimisation has been described in e.g. Amiri-Simkooei (2003). Following this general formulation, in order to deal with absolute value function in the Equation 2, we re-write the vectors $\mathbf{v}$ and $\mathbf{x}$ with the help of slack variables. This will reduce the problem to that of a linear programming. These vectors are now given by

$$
\begin{aligned}
& \mathbf{v}=\mathbf{u}-\mathbf{w}, \quad \mathbf{u}, \mathbf{w} \geq 0 \\
& \mathbf{x}=\boldsymbol{\alpha}-\boldsymbol{\beta}, \quad \boldsymbol{\alpha}, \boldsymbol{\beta} \geq 0
\end{aligned}
$$

where a condition $u_{i}$ or $w_{i}=0$ holds for the residual vector components. Now given the conditions in Equation 7a, Equation 2 can be written as

$$
\mathbf{p}^{\top}|\mathbf{v}|=\mathbf{p}^{\top}|\mathbf{u}-\mathbf{w}|=\mathbf{p}^{\top}(\mathbf{u}+\mathbf{w})
$$

subject to the conditions in Equation 7b, 
190 The objective function can now be written as

191

$193+\left[\begin{array}{llll}\mathbf{A} & -\mathbf{A} & \mathbf{I} & -\mathbf{I}\end{array}\right]\left[\begin{array}{l}\alpha \\ \beta \\ \mathbf{w} \\ \mathbf{u}\end{array}\right]=\mathbf{y}$,

192

194 given the same conditions as earlier. Denoting the objective function with $195 z$ this form is equivalent to

$$
\min \left(\left[\begin{array}{llll}
\mathbf{0}^{\mathbf{T}} & \mathbf{0}^{\mathbf{T}} & \mathbf{p}^{\mathbf{T}} & \mathbf{p}^{\mathbf{T}}
\end{array}\right]\left[\begin{array}{l}
\alpha \\
\beta \\
\mathbf{w} \\
\mathbf{u}
\end{array}\right]\right)
$$

subject to

$$
z=\mathbf{c}^{\top} \mathbf{x},
$$

$$
\mathrm{Ax}=\mathrm{b}, \quad \mathrm{x} \geq 0
$$

The L1-norm minimisation was implemented in c5++ as an external python script. The corresponding linear programming problem was solved using a Simplex-method (Murty, 1983) implemented in the linprog function of the optimisation module in SciPy (Walt et al., 2011). 


\subsection{Theoretical comparison of L1- and L2-norm}

Generally, the advantage of the L2-norm is that it is computationally simple. The L2-norm is intrinsically stable and it has a unique solution. Furthermore, if the measurement errors are assumed to be normally distributed around 0 with a variance of $\sigma^{2}$, the L2-norm is the maximum likelihood estimator (MLE) for the unknowns. Thus, for normally distributed errors, the L2-norm will give the optimal estimates for the unknowns. However, assuming normality might not always be justified, and it can be hard to infer from the results whether the assumption was in fact correct. The sampling variance of the L2-norm is proportional to $\sigma^{2} / n$, where $n$ is the sample size. Even though the L2-norm is efficient, its disadvantage is its sensitivity to outliers. Because the sum deals with squared residuals, large deviations in the residuals tend to have high impact on the overall sum. This in turn will propagate into the unknowns.

Compared to the L2-norm, the main advantage of the L1-norm is its increased robustness against outliers. Since the L1-norm sums absolute deviations instead of squared values, large residuals do not influence the solution to the same degree as with the L2-norm. Consequently, the L1-norm will more likely correctly detect the magnitude of the large outliers, instead of propagating the error into the unknowns through the adjustment. The L2norm tends to overcompensate the influence of large deviations. For example, in case of a simple linear regression, by shifting the regression line towards the outliers, making the individual residual smaller but consequently providing a worse fit for the good observations. With the L1-norm large deviations do not dominate the sum to a same degree, and thus in case of e.g. linear 
regression, the fit is not shifted towards the erroneous observation as much, keeping the residuals of the good observations smaller and correctly detecting the magnitude of the bad one.

However, the L1-norm has some clear disadvantages compared to the L2-norm, as well. Firstly, the solution is not always stable and there is no guarantee of a unique solution. In contrast to the MLE condition of the L2-norm, the L1-norm is the MLE when the errors follow a Laplace distribution with $\mu$ and $b$ as the location and scale parameters. In case of normally distributed errors $e \sim \mathrm{N}\left(0, \sigma^{2}\right)$ the sampling variance for L1-norm is proportional to $(\pi / 2)\left(\sigma^{2} / n\right)$ (Andersen, 2008). Thus, if the errors are in general normally distributed, the L1-norm will likely produce larger variance compared to the L2-norm.

Keeping these considerations in mind, the L1-norm has the potential to be very effective in detecting outlier of large magnitude. This corresponds well to the case of the ambiguity resolution problem in geodetic VLBI where the ambiguities have far greater magnitude than the overall noise-floor of the observations.

\section{Automated ambiguity estimation for Intensive sessions}

To investigate the performance of the L1-norm in the ambiguity estimation we analysed a total of 1835 INT1 sessions observed in the period of 2001-2015, starting from Version-1 databases. The sessions were analysed in automated mode by resolving the ambiguities with both the L1- and L2norm approach. The ambiguity-resolved databases were then subsequently processed to estimate the UT1-UTC with respect to the EOP product of 
International Earth Rotation and Reference Frame Service (IERS), namely EOP C04 08 (Bizouard and Gambis, 2011). This latter estimation was carried out using the standard L2-norm method for both ambiguity resolving methods. Thus, the only differences to the analysis due to the L1- and L2norms are introduced in the ambiguity estimation step.

\subsection{Ambiguity estimation in $c 5++$}

The general ambiguity estimation process in $\mathrm{c} 5++$ is iterative. The $\mathrm{X}$ and S-band group delays are processed as independent observations, which retains the integer-nature of the ambiguities. In contrast, the software package SOLVE (Ma et al., 1990), which has long been used operatively for the IVS data products, combines the $\mathrm{X}$ - and S-band group delays in the initial stage of the automated ambiguity estimation. The modern replacement for Solve, vSolve (Bolotin et al., 2014), implements similar concepts in its automated group delay ambiguity estimation.

The functional model used for the ambiguity resolution in $\mathrm{c} 5++$ is described in Hobiger et al. (2010). In general, the model includes a quadratic polynomial for the station clock behaviour, an offset term between X-and S-band to consider inter-band instrumental delays, and the troposphere delays at each station. One of the stations is always chosen as the reference, for which the clock and inter-band offsets are not estimated. Thus, in the case of INT1 and one baseline we estimate in total three clock coefficients and the band offset term for the non-reference station. In this analysis the troposphere parameters were also estimated. The troposphere parameters at the stations are estimated to the zenith-direction and mapped to the source elevation using a mapping function. There are multiple mapping functions 
available. In this analysis we used the Global Mapping Functions (GMF2) together with Global Pressure and Temperature Model (GPT2) (Lagler et al., 2013).

In each iteration step the residuals are computed and if they are larger than $50 \%$ of the ambiguity spacing on that band, the corresponding observations are shifted by one ambiguity spacing towards 0 . This process of ambiguity shifting is iterated until the ratio of the WRMS values of the residuals from subsequent iterations reaches a pre-specified level. This level was set to 0.999 in all our analyses which are discussed hereafter. The maximum number of iterations was set to 60 . During the estimation process, different weighting schemes can be applied. The effect of the choice of weighting was investigated using three different approaches, which are described in Table 1.

Table 1: The three different weighting approaches used in the ambiguity estimation.

\begin{tabular}{lll}
\hline $\begin{array}{l}\text { Weighting } \\
\text { mode }\end{array}$ & Description & Weighting \\
\hline $\mathbf{W} \mathbf{1}$ & Unit weighting & \\
$\mathbf{W} 2$ & Formal errors & $\frac{1}{\sigma_{\tau}^{2}}$ \\
$\mathbf{W} 3$ & Formal errors multiplied by & $\frac{1}{\sigma_{\tau}^{2}\left(\operatorname{mf}(e)_{\text {wet }, 1}^{2}+\operatorname{mf}(e)_{\text {wet }, 2}^{2}\right)}$ \\
& wet mapping functions val- & \\
& ues (elevation dependent) & \\
\hline
\end{tabular}

Once the ambiguities are resolved, the X- and S-band data are combined to produce an ionosphere free X-band database. This database is then sub- 
sequently used as an input in the UT1-UTC estimation step. In this step the observations were weighted according to the elevation dependent approach, W3. The schematics in Figure 1 illustrate the ambiguity and UT1-UTC estimation process in $\mathrm{c} 5++$.

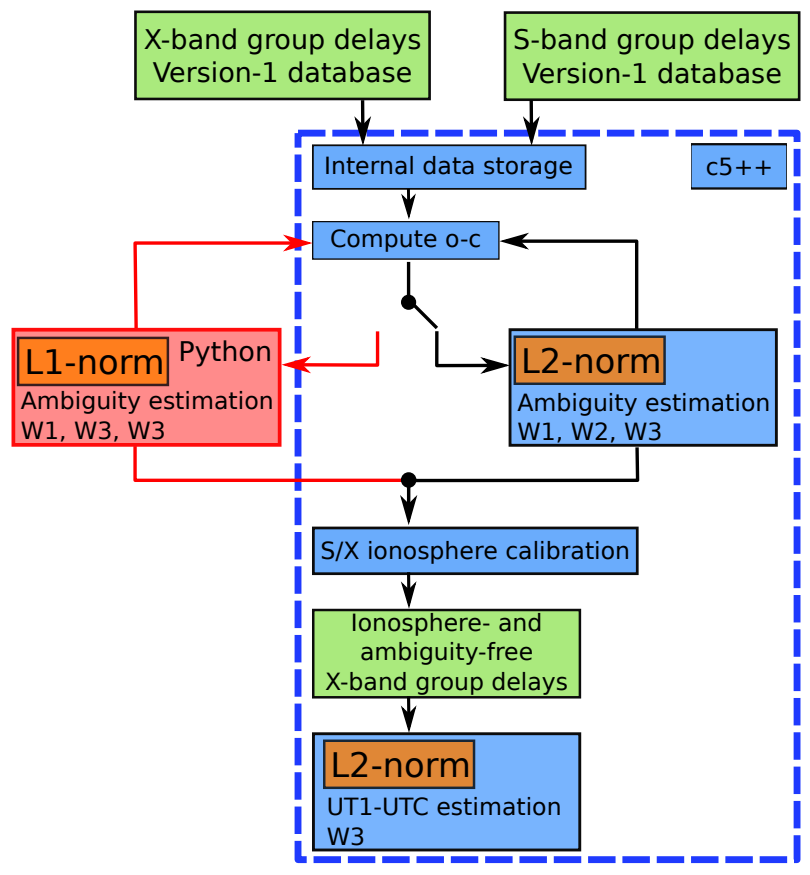

Figure 1: Schematics of the automated ambiguity and UT1-UTC estimation in c5++.

\subsection{Indicators for successfully resolved ambiguities}

In order to assess whether the ambiguities have indeed been successfully resolved, we need to define criteria, that capture the effect of the ambiguity estimation.

Since any unresolved ambiguities will propagate into the estimated parameters, a straightforward method is to investigate the UT1-UTC estimates obtained from the two ambiguity estimation approaches. In this estimation 
step the station coordinates were kept fixed to their a priori ITRF2008 (Altamimi et al., 2011) values. Except UT1-UTC, all EOP were fixed to their EOP C04 08 values. The UT1-UTC were estimated with respect to the a priori C04 08 values. From now on these values will be referenced simply as the UT1-UTC estimates. In addition to UT1-UTC, a quadratic clock for the non-reference stations and the wet troposphere for both stations were estimated. This set-up is typical for INT sessions, since due to the combination of short session duration and baseline geometry, the only EOP that can be viably determined is UT1-UTC.

Furthermore, we can directly compare the Root Mean Square (RMS) and Weighted RMS (WRMS) values for the post-fit residuals from the ambiguity resolution runs. In the ambiguity resolution step no outlier elimination is performed, because in the presence of ambiguities every observation has the potential to be interpreted as an outlier. Thus, any unresolved ambiguities will be reflected as higher RMS and WRMS values. In the UT1-UTC estimation step in $\mathrm{c} 5++, 3-\sigma$ outliers are detected at iteration step $i$ following an empirically derived condition (Hobiger et al., 2010)

$$
|o-c|_{i}>\mathrm{WRMS}_{i-1} \frac{3}{\sqrt{2}} \sqrt{\operatorname{mf}(e)_{\text {wet }, 1}^{2}+\operatorname{mf}(e)_{\text {wet }, 2}^{2}} \Rightarrow \text { outlier, }
$$

where the $o-c$ is the difference between the observed and computed value at the $i$ th iteration, $\mathrm{WRMS}_{i-1}$ is the WRMS value from the previous iteration, and $\mathrm{mf}_{\text {wet, } 1}^{2}$ and $\mathrm{mf}_{\text {wet, } 2}^{2}$ are the wet mapping function values for the stations 1 and 2, respectively. This outlier detection algorithm implies that if the WRMS from the previous iteration is high, the solution is more tolerant to large residuals. 


\section{Results}

The impact of using the L1-norm in the ambiguity estimation was examined by investigating the criteria described in Section 3.2. In order to focus on the sessions which produced meaningful UT1-UTC estimates w.r.t C04 and to eliminate gross errors that would distort the derived statistics of the UT1-UTC, the estimates, meaning the adjustments to the a priori values, were filtered with a condition where the absolute values of the estimates are larger than $1000 \mu \mathrm{s}$ and/or the formal errors are larger than $50 \mu \mathrm{s}$. After this initial outlier elimination was applied, we obtained a set of sessions for each ambiguity estimation method-weighting mode pair, for which RMS and WRMS of the UT1-UTC values were computed. These values are listed in Table 2. The largest number of good sessions is highlighted for each weighting strategy. The RMS and WRMS values for the post-fit residuals from the ambiguity estimation for both norms and all weighting strategies are listed in Table 3.

The results in Table 2 show that the RMS and WRMS values of the UT1-UTC estimates for both approaches are very close. The differences in all categories are below $0.2 \mu$ s. These values reflect the general level of UT1-UTC accuracy obtainable from INT sessions (Kareinen et al., 2015). The noteworthy conclusion is that the L1-norm gives a larger number of good sessions compared to the L2-norm, and this is true for all weighting strategies. The largest difference is seen with weighting W1, where the L1norm approach to resolve ambiguities produces 84 more good sessions. The L1-norm resulted in an increase of $5.4 \%, 4.4 \%$, and $4.6 \%$ for the number of good sessions using the W1, W2, and W3 weightings, respectively. 
Table 2: Impact of the weighting strategies for ambiguity estimation on final UT1-UTC results. Presented are the number of sessions and corresponding RMS/WRMS of UT1-UTC values w.r.t. $\mathrm{C} 04$ for the sessions, which pass the $|\mathrm{UT} 1-\mathrm{UTC}|<1000 \mu \mathrm{s}$ and $\sigma_{\mathrm{UT} 1-\mathrm{UTC}}$ $<50 \mu$ s criteria. For each weighting strategy the highest number of sessions between the L1- and L2-norm approaches are highlighted in boldface.

\begin{tabular}{|c|c|c|c|c|c|c|}
\hline & \multicolumn{3}{|c|}{ L1 } & \multicolumn{3}{|c|}{$\mathrm{L} 2$} \\
\hline & \#Sessions & $\mathrm{RMS}[\mu \mathrm{s}]$ & WRMS $[\mu \mathrm{s}]$ & \#Sessions & $\mathrm{RMS}[\mu \mathrm{s}]$ & WRMS $[\mu \mathrm{s}]$ \\
\hline W1 & 1649 & 22.58 & 18.39 & 1565 & 22.58 & 18.37 \\
\hline W2 & 1469 & 22.32 & 18.43 & 1407 & 22.50 & 18.53 \\
\hline W3 & 1493 & 22.25 & 18.43 & 1428 & 22.42 & 18.37 \\
\hline
\end{tabular}

Table 3: Mean RMS and WRMS of the post-fit residuals from the ambiguity estimation for L1- and L2-norms for all weighting strategies.

\begin{tabular}{cccccc}
\hline & \multicolumn{2}{c}{ L1 } & & \multicolumn{2}{c}{$\mathrm{L} 2$} \\
\cline { 5 - 6 } \cline { 5 - 5 } & $\overline{\text { RMS }[\mathrm{m}]}$ & $\overline{\text { WRMS }[\mathrm{m}]}$ & & $\overline{\mathrm{RMS}}[\mathrm{m}]$ & $\overline{\mathrm{WRMS}}[\mathrm{m}]$ \\
\hline $\mathbf{W 1}$ & 1.08 & 1.08 & & 1.25 & 1.25 \\
$\mathbf{W 2}$ & 1.87 & 0.42 & & 1.86 & 0.51 \\
$\mathbf{W 3}$ & 1.83 & 0.37 & & 1.87 & 0.43 \\
\hline
\end{tabular}


The mean RMS and WRMS of the post-fit residuals presented in Table 3 show that the L1-norm gives on average a better fit after the ambiguity estimation. The only exception is weighting W2 where the RMS of the L2norm is smaller by 30 ps.

The number of iterations that it takes for the ambiguity estimation to converge may reflect both the initial quality of the data, the impact of the weighting method, as well as the stability of the estimation method. The number of iterations for the L1- and L2-norms approaches and weightings W1, W2, and W3 are presented in Figure 2.

The success of the ambiguity estimation is reflected in the post-fit group delay residuals from the UT1-UTC estimation. The errors from the unresolved ambiguities propagate to the estimated parameters during the first iteration of the UT1-UTC estimation. The outlier elimination algorithm in c5++ given in Equation 14 depends on the WRMS of the previous iteration. Parameters estimated in the first iteration bear the risk to absorb outliers and thus subsequent iterations are not able discern between good observations and outliers.

Shown in Figure 3 are the residuals for the both L1- and L2-norm approaches and all three weighting strategies. It becomes clear that the residuals from the L1-norm approach are smaller in general. This can be confirmed both with more L1-residuals located closer to zero and less L1-residuals with large magnitudes.

The overlap of the sets of good sessions obtained with the L1- and L2norm approaches are illustrated by Venn-diagrams in Figure 4. These diagrams show the number of good sessions which are found in both L1 and L2, 


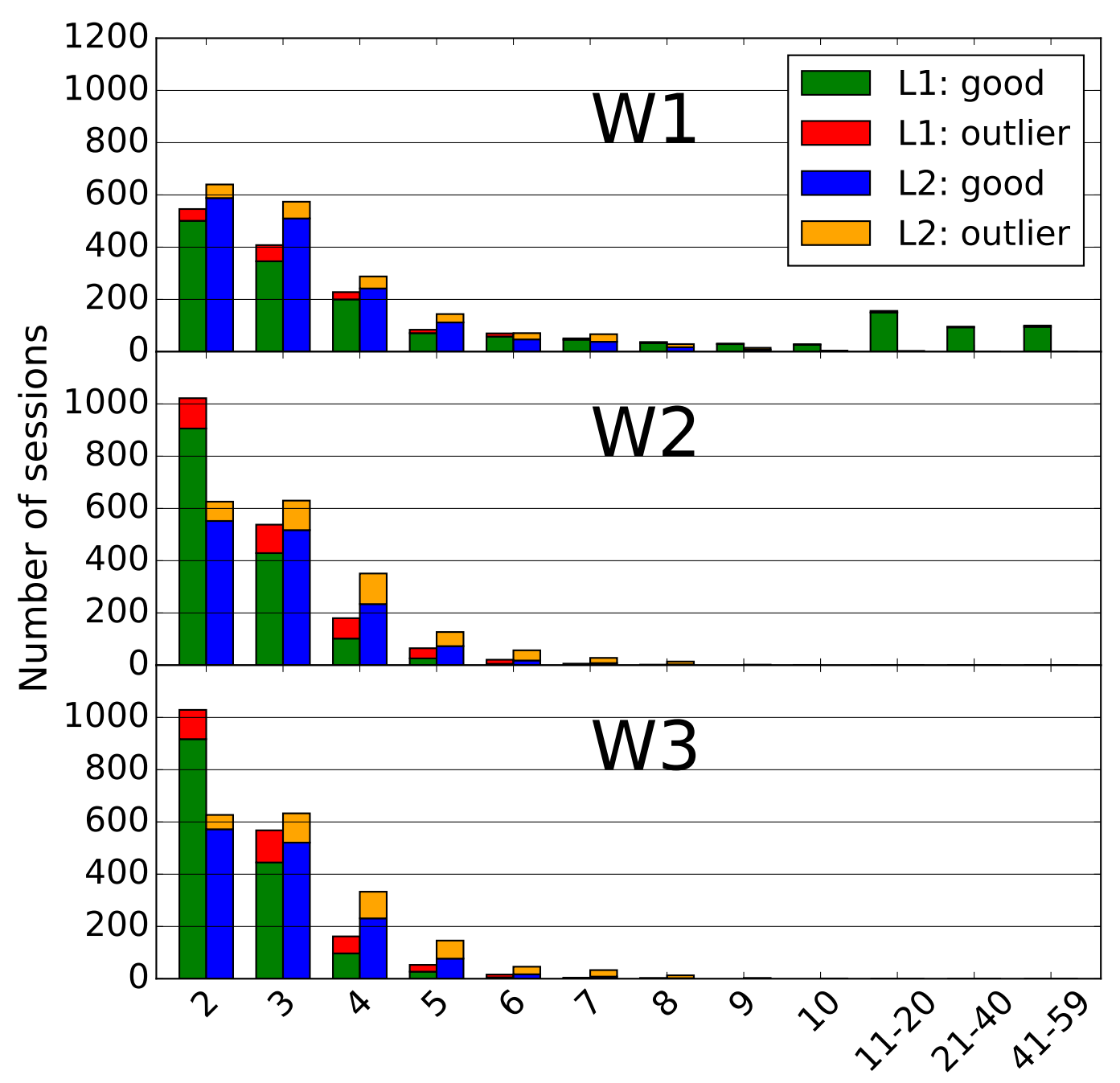

Number of iterations

Figure 2: Session distribution by the number of iterations for the L1- and L2-norm approaches for each weighting strategy W1, W2, and W3. The histograms separate between the sessions passing the $1000 \mu \mathrm{s} / 50 \mu \mathrm{s}$ criterion with a different pair of colours for both norms.

only L1, or only L2 results.

To investigate the sessions that fail with either the L1- or L2-norm ap- 

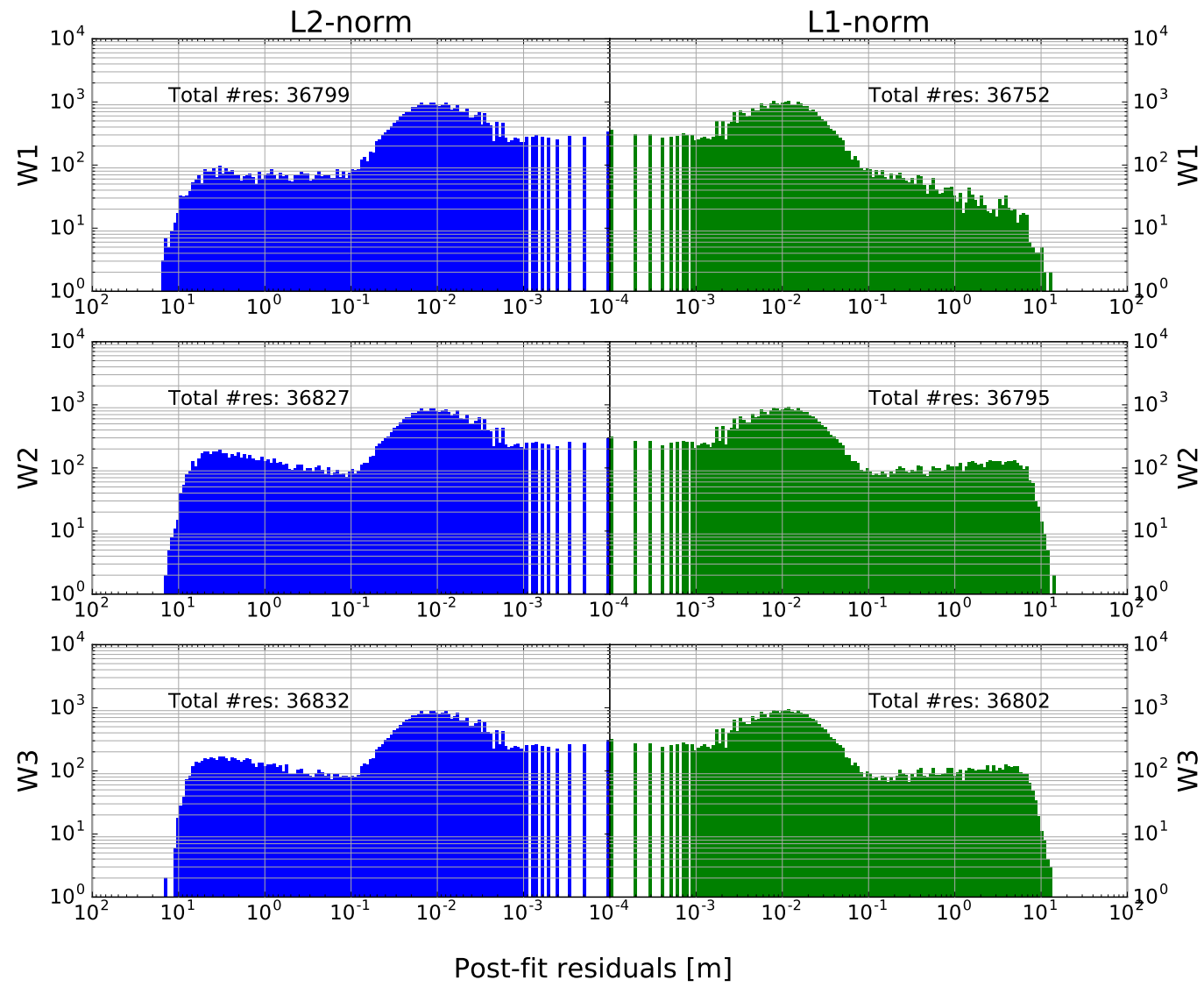

Figure 3: The distribution of the post-fit residuals from UT1-UTC estimation for the L2(left, blue) and L1-norm (right, green) approaches and weighting strategies W1, W2, and W3.

proaches, we consider subsets from all the sessions that resulted in good

- Subset-1: select all sessions that are good with the L1-norm approach, select the same sessions from the L2-norm solutions. 


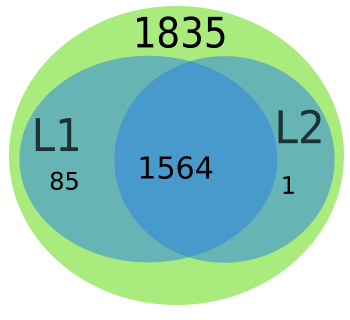

(a) Weighting: W1

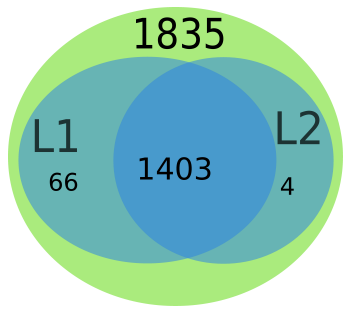

(b) Weighting: W2

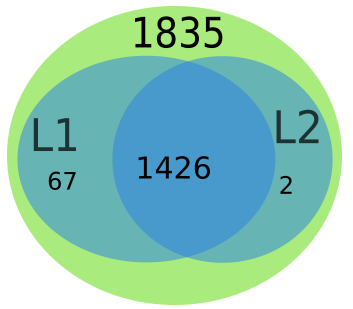

(c) Weighting: W3

Figure 4: Venn-diagrams for the weighting strategies W1, W2, and W3 illustrating the overlap between the sets of sessions obtained with the L1- and L2-norm ambiguity estimation, that pass the $|\mathrm{UT} 1-\mathrm{UTC}|<1000 \mu$ s and $\sigma_{\mathrm{UT} 1-\mathrm{UTC}}<50 \mu$ s criteria.

- W1: $1564+85=1649$ sessions

$-\mathrm{W} 2: 1403+66=1469$ sessions

- W3: $1426+67=1493$ sessions

- Subset-2: select all sessions that are exclusively good with the L1-norm approach, select the same sessions from the L2-norm solutions.

- W1: 85 sessions

- W2: 66 sessions

- W3: 56 sessions

- Subset-3: select all sessions that are exclusively good in the L2-norm approach, select the same sessions from the L1-norm solutions.

- W1: 1 session

- W2: 4 sessions

- W3: 2 sessions 
The average number of observations (X- or S-band) in the whole set of 1835 sessions is 20.5 . For the Subset-1 and weighting strategies W1, W2, and W3, the average number of observations are 20.7, 20.8, and 20.8. These values are slightly higher the average number observations of all sessions. This shows that on average sessions with a higher number of observations lead to better UT1-UTC estimate.

Similarly, for the Subset-2 the average number of observations are 18.8, 20.6, and 20.4. For weighting W2 and W3 the average number of observations between Subset 2 and all sessions are very close to one another. For the weighting W1 the larger number of extra sessions between L1- and L2-norm compared to weightings W2 and W3 correspond to including sessions that have less than average number of observations when compared to the average of all sessions. Thus, with weighting W1 the L2-norm approach fails more often than the L1-norm approach with sessions that had slightly less than average number of observations.

The Subset-3 has very few observations in all the weighting strategies W1, W2, and W3. The average number of observations in Subset-3 and weightings $\mathrm{W} 1, \mathrm{~W} 2$, and $\mathrm{W} 3$, are 16.0, 20.3, and 20.5, respectively. These values are similar to the corresponding Subset-2 results. This indicates that the failure of the L1-norm approach in Subset-3 is not related to the number of observations in these sessions. Furthermore, weighting W1 again has lower than average number of observations. Based on the number of observations in Subset-2 and Subset-3 with weighting W1 the low number of observations cause instability, which the L1-norm approach is able to handle better.

Next, we investigate the extent to which the added sessions obtained 
with the L1-norm approach influences the UT1-UTC accuracy by selecting the sessions in Subset-1. The results from this comparison are presented in Table 4. The counterpart to the Subset-1 would be a subset, where we select the good L2-norm sessions and the same sessions processed with the L1-norm. However, since there are only a few sessions that have a good UT1-UTC solution exclusively with the L2-norm, their relative number with respect to the total number of good sessions is very low. Thus it is not meaningful to consider the RMS/WRMS of the UT1-UTC estimates based on these sessions. This is also the case for the Subset-3. In the following we focus only on Subset-1 and Subset-2.

Table 4: Number of sessions and corresponding RMS/WRMS of UT1-UTC values w.r.t. C04 for the sessions included in Subset-1.

\begin{tabular}{ccccccc}
\hline & & \multicolumn{2}{c}{ L1 } & & \multicolumn{2}{c}{ L2 } \\
\cline { 7 - 7 } \cline { 6 - 7 } \cline { 6 - 7 } \#Sessions & RMS $[\mu \mathrm{s}]$ & WRMS $[\mu \mathrm{s}]$ & & RMS $[\mu \mathrm{s}]$ & WRMS $[\mu \mathrm{s}]$ \\
\hline W1 & 1649 & 22.58 & 18.39 & & 938.09 & 18.70 \\
W2 & 1469 & 22.32 & 18.43 & & 805.21 & 18.82 \\
W3 & 1493 & 22.25 & 18.43 & & 1096.07 & 18.74 \\
\hline
\end{tabular}

32 The number of extra sessions obtained with the L1-norm is approximately $5 \%$ compared to the L2-norm. The large increase in the RMS values compared to the WRMS values in the L2-norm indicate that the sessions previously discarded due to high UT1-UTC estimate have correspondingly large 
Table 5: Number of sessions and corresponding RMS/WRMS of UT1-UTC values w.r.t. C04 for the sessions included in Subset-2.

\begin{tabular}{|c|c|c|c|c|c|}
\hline & \multirow[b]{2}{*}{ \#Sessions } & \multicolumn{2}{|r|}{ L1 } & \multicolumn{2}{|r|}{$\mathrm{L} 2$} \\
\hline & & $\operatorname{RMS}[\mu s]$ & WRMS $[\mu \mathrm{s}]$ & $\mathrm{RMS}[\mu \mathrm{s}]$ & WRMS $[\mu \mathrm{s}$ \\
\hline W1 & 85 & 18.83 & 22.54 & 3934.66 & 4130.73 \\
\hline W2 & 66 & 17.11 & 19.38 & 3280.11 & 3797.42 \\
\hline W3 & 67 & 19.48 & 19.55 & 2646.68 & 5173.04 \\
\hline
\end{tabular}

formal errors. Thus, they are heavily downweighted in the WRMS of the UT1-UTC for all three weighting strategies. Comparing the results from the L2-norm approach presented in Tables 4 and 2 one can see that the WRMS values for the L2-norm in Subset-1 are slightly larger.

Overall, the greatest contribution of the L1-norm approach is the number of added sessions, which increase the time resolution of the UT1-UTC series, rather than the overall accuracy.

When we investigate the set of sessions, which pass the outlier filtering only in the L1-norm approach (see Figure 4), we see a clear difference both in RMS and WRMS values between the two norms. These values are listed in Table 5. Now the L2-norm approach produces large values in both RMS and WRMS values. These WRMS values indicate, that the formal errors of the UT1-UTC estimates for the extra sessions in the L2-norm have similar magnitude. 


\section{Conclusions}

The increased number of sessions that produce good quality UT1-UTC estimates indicate that the L1-norm clearly improves the automated ambiguity estimation for the INT1 sessions. The improvement provided by the L1-norm is also supported by the generally smaller RMS and WRMS values of the post-fit residuals from the ambiguity estimation. In general the L1norm approach yields an improvement of 15-20\% in WRMS of the post-fit residuals. The subset of added sessions with respect to the L2-norm approach generally represent an average sample of INT1 sessions. The average number of observations in the sessions which benefited from the L1-norm ambiguity estimation is almost identical to the average number of observations over the whole set of analysed INT1 sessions. This implies that the improvement in ambiguity resolution with the L1-norm is not correlated with particularly high or low number of observations in the sessions.

The number of sessions that are improved by the L1-norm approach greatly outnumber the ones where the issues of stability result in a failed ambiguity estimation. Quantitatively, the increase in number of sessions by using the L1-norm is approximately $5 \%$.

The computational complexity of solving the linear programming problem compared to inverting the normal equations does not generally cause significant overhead in the processing time for an individual session. The convergence of the L1-norm varied between the different weighting approaches. For the L2-norm the different weighting options behaved more uniformly. However, slow convergence does not necessarily lead to bad quality of the results. Using the W1 weighting, the L1-norm iteration counts were signif- 
475

icantly larger compared to those of the L2-norm. However, the L1-norm using the W1 weighting (i.e. equally weighted) produced the biggest increase in good quality UT1-UTC estimates.

\section{Acknowledgements}

We would like to acknowledge the IVS (Behrend, 2013) for providing the VLBI databases used in this work. We also would like to thank the two anonymous reviewers for their valuable comments that helped greatly to improve this paper.

\section{References}

Altamimi, Z., Collilieux, X., Métivier, L., 2011. ITRF2008: an improved solution of the international terrestrial reference frame. J. Geod. 85 (8), $457-473$.

Amiri-Simkooei, A. R., 2003. Formulation of $L_{1}$ norm minimization in GaussMarkov models. J. Surv. Eng. 129 (1), 37-43.

Andersen, P., 2000. Multi-level arc combination with stochastic parameters. J. Geod. 74 (7-8), 531-551.

Andersen, R., 2008. Modern methods for robust regression. No. 152 in 7. Sage.

Barnes, R. T. H., Hide, R., White, A. A., Wilson, C. A., 1983. Atmospheric angular momentum fluctuations, length-of-day changes and polar motion. Proc. R. Soc. London, Ser. A 387 (1792), 31-73. 
Behrend, D., 2013. Data Handling within the International VLBI Service. Data Sci. J. 12, WDS81-WDS84.

Bizouard, C., Gambis, D., 2011. IERS C04 08. https://hpiers.obspm.fr/iers/eop/eopc04/C04.guide.pdf, the combined solution C04 for Earth Orientation Parameters consistent with International Terrestrial Reference Frame 2008. Accessed: 2016-06-29.

Böhm, J., Böhm, S., Nilsson, T., Pany, A., Plank, L., Spicakova, H., Teke, K., Schuh, H., 2012. 'The new Vienna VLBI software VieVS'. In: Kenyon, S., Pacino, M. C., Marti, U. (Eds.), Geodesy for Planet Earth: Proceedings of the 2009 IAG Symposium. Vol. 136. Springer, pp. 1007-1011.

Bolotin, S., Baver, K., Gipson, J., Gordon, D., MacMillan, D., 2014. 'The VLBI Data Analysis Software $\nu$ Solve: Development Progress and Plans for the Future'. In: Baver, K. D., Behrend, D., Armstrong, K. L. (Eds.), IVS 2014 General Meeting Proceedings "VGOS: The New VLBI Network". Science Press, Beijing, pp. 253-257.

Brzeziński, A., 2012. On estimation of high frequency geophysical signals in Earth rotation by complex demodulation. J. Geodyn. 62, 74-82.

Hobiger, T., Otsubo, T., Sekido, M., Gotoh, T., Kubooka, T., Takiguchi, H., 2010. Fully automated VLBI analysis with $\mathrm{c} 5++$ for ultra-rapid determination of UT1. Earth Planets Space 62 (12), 933-937.

Kareinen, N., Hobiger, T., Haas, R., 2015. Automated analysis of KokeeWettzell Intensive VLBI sessions - algorithms, results, and recommendations. Earth Planets Space 67, 181. 
Koch, K.-R., 1999. Parameter estimation and hypothesis testing in linear models. Springer-Verlag Berlin Heidelberg.

Lagler, K., Schindelegger, M., Böhm, J., Krasna, H., Nilsson, T., 2013. GPT2: Empirical slant delay model for radio space geodetic techniques. Geophys. Res. Lett. Vol. 40 (6), 1069-1073.

Ma, C., Sauber J.M., Bell L.J., Clark, T., Gordon, D., Himwich W.E., Ryan J.W., 1990. Measurement of horizontal motions in Alaska using very long baseline interferometry. J. Geophys. Res. 95 (B13), 21991-22011.

Malkin, Z., Skurikhina, E., 2005. OCCAM/GROSS Software Used at the IAA EOP Service for processing of VLBI Observations. Transactions of the Institute of Applied Astronomy of the RAS 12, 54-67.

Murty, K. G., 1983. Linear programming. John Wiley \& Sons.

Rogers, A. E., 1970. Very long baseline interferometry with large effective bandwidth for phase-delay measurements. Radio Sci. 5 (10), 1239-1247.

Titov, O., Tesmer, V., Böhm, J., 2004. 'OCCAM v. 6.0 software for VLBI data analysis'. In: Vanderberg, N. R., Baver, K. D. (Eds.), IVS 2004 General Meeting Proceedings. NASA/CP-2004-212255, pp. 267-271.

Titov, O., Tregoning, P., 2005. Effect of post-seismic deformation on earth orientation parameter estimates from VLBI observations: a case study at Gilcreek, Alaska. J. Geod. 79, 196-202.

Walt, S., Colbert, S. C., Varoquaux, G., 2011. The NumPy Array: A Structure for Efficient Numerical Computation. Comput. Sci. Eng. 13 (2), 22-30. 